\title{
XERODERMA PIGMENTOSUM
}

\author{
Presentación de un caso y revlsión de la literatura.
}

\author{
Por el Dr. DARIO SepUl VedA R.
}

Xeroderma pigmentosum es una enfermedad rara, cuyos rasgos característicos lo constituyen manchas pigmentarias. cicatriciales y telangectasias en una piel xerodérmica, a la cual se agregan progresivamente elementos verrucosos de tendencia degenerativa.

Como sucedo con la mayoría de las dermatosis, ha recibido múltiples denominaciones: Xeroderma (Hebra y Kaposi), Nevus de forma rara (Geeber), Angioma pigmentario y atrófico (Taylor), Liodermia esencial con melanosis y telangectasias (Neisser), Melanosis lenticular progresiva (Pick), Atrophoderma pigmentosum (Crocker), Lentigo epiteliomatoso (Quinquaud), Pigmentosis epitelial (Besnier), Enfermedad de Kaposi (Vidal).

Es una enfermedad probablemente congénita, familiar, transmitida con carácter recesivo. Cuando la enfermedad aparece en los hijos de patres consanguíreos, el número de afectados sería mayor que en el caso en que los padtes no son parientes entre sí. Ambos sexos son igualmente afectados. Más frecuente en la raza judía. Annquo su etiología exacta es desconocida, hay una aparente hipersensibilidad de la piel a los rayos luminosos de onda largas. En gran número de casos se encuentra una tríada predisponente caracterizada por: piel muy blanca, cabcllos rubios y ojos azules.

Aparece, por lo común, en el primer año de la vida; aunque las formas abortivas no se hacen aparentes hasta más tarde. Las lesiones son más marcadas en las zonas cutáncas expuestas a la luz: cara, cuello, manos, antebrazos y pietnas. Sus elementos están formados por: manchas pigmentarias como las pecas o lentigos: manchas leucodérmicas y atróficas que producen, a nivel de los párpados, ectropión: telangectasias, especialmente alrededor de aquellas últimas; excreciones verrucosas semejantes a las verrugas seniles $y$ que terminan por exfoliarse o transformarse en un tumor maligno; ulceraciones superficiales, especialmente a nivel de la cara, y finalmente, epiteliomas basocelulares o melanomas.

La evolución se hace en tres períodos: 1) En un primer período se trata sintplemonte de una eritrodermia, que aparece cuando el niño comienza a salir fuera de la casa. La piel toma un aspecto seco, jaspeado, áspero, con marcada hiperemia capilar difusa, especialmente a nivel de la cara.

2) Ya al tercero o cuarto año de edad, la pigmentación es más notoria; hay descamación furfurácea y aparecen las excrecencias verrugosas.

3) En un tercer estado, se inicia la degeneración con manchas lélicodérmicas atróficas, entro las que sc entremezclan telangectasias y aumentan las lesiones verrugosas, que toman un carácter malig$110 \mathrm{y}$ se ulceran.

El curso de la enfernedad es progresivo: pero es cotriente que nuestro períodos de inactividad. Lo común es que la sobrevida no pase de $\log 21$ años de edad, ocurriendo la muerte, generalmente, por caquexia. 
La literalura mundial hasta el año tratados clásicos de dermatología no men1930 anetaba 80 casos $^{4}$, la mayoria pliblicados par dermatólogos. Desde el año 1930, en la literatura oxtrav jera a nuestre alcance, se mencionan uuy pocos casos y en la nacional sólo hemos encontrado 3 observalos simultáneamente por Morales Villablanca en ese año". Los cionan la deficiencia mental y ciertas alteraciones nervionas como una parte dol cuatro clínico del xeroderma pigmentosim. al paso que han siclo señalarlas por varios pediatrañ 1311 . Estos casos se encucntran resusuidos en el cuadro.

Casos de Xeroderma Pigmentusum en la literalura desde 1930.

\begin{tabular}{|c|c|c|c|c|}
\hline Autor & Edad & $\begin{array}{l}\text { Consan- } \\
\text { guinidad }\end{array}$ & $\begin{array}{l}\text { Erdad de } \\
\text { comienzo }\end{array}$ & Evolucion \\
\hline $\begin{array}{l}\text { Morales V." } \\
3 \text { casos }\end{array}$ & $\begin{array}{l}5 \text { años } \\
4 \text { años } \\
5 \text { años }\end{array}$ & $\begin{array}{l}\text { positivo } \\
\text { hermaino } \\
\text { positivo }\end{array}$ & $\begin{array}{l}\mathbf{3} \text { meses } \\
\mathbf{5} \text { meses } \\
1 \text { año }\end{array}$ & $\begin{array}{l}\text { no consignada } \\
\text { no consignada } \\
\text { no consignada }\end{array}$ \\
\hline Blackmar ${ }^{\$}$ & 6 años & $?$ & 6 meses & no consignada \\
\hline Foldvari 7 & $?$ & $?$ & $?$ & $\begin{array}{l}\text { Muerte a los } 9 \text { años de edad. La autog- } \\
\text { sia reveló epitelioma que tomaba el co- } \\
\text { sium, un sarcoma del tejido celuiar sulh- } \\
\text { cutáneo y un melanosarcoma metastá- } \\
\text { sico del higado. }\end{array}$ \\
\hline $\begin{array}{l}\text { Belisario: } \\
2 \text { casos }\end{array}$ & $\begin{array}{r}8 \text { años } \\
14 \text { años }\end{array}$ & $\begin{array}{l}\text { negativo } \\
\text { negativo }\end{array}$ & $\begin{array}{l}6 \text { meses } \\
3 \text { meses }\end{array}$ & $\begin{array}{l}\text { A los } 3 \text { años de edad parálisis de la mu- } \\
\text { neca izquienda, junto con pérdida par- } \\
\text { cial de la función de la mano y dedos. }\end{array}$ \\
\hline $\begin{array}{l}\text { Bell } \\
2 \text { casos }\end{array}$ & $\begin{array}{l}16 \text { años } \\
13 \text { años }\end{array}$ & $\begin{array}{l}\text { positivo } \\
\text { positivo }\end{array}$ & $?$ & $\begin{array}{l}\text { Epitelioma del labio inferior en los } 2 \\
\text { hermanos, curados con radioterapia in- } \\
\text { tensiva del labio y ganglios. }\end{array}$ \\
\hline Zienkiewicz"14 & 8 años & positivo & 9 moses & no consignada \\
\hline $\begin{array}{l}\text { Silberstein }{ }^{13} \\
2 \text { casos }\end{array}$ & $\begin{array}{l}13 \text { años } \\
12 \text { años }\end{array}$ & $\begin{array}{l}\text { negativo } \\
\text { negativo }\end{array}$ & $\begin{array}{l}1 \text { año } \\
6 \text { meses }\end{array}$ & $\begin{array}{l}\text { En ambos hermanos retardo mental, } \\
\text { alteracicnes de la marcha y del lent- } \\
\text { guaje. El 1. con retando osifícación del } \\
\text { carpo tedad ósea } 10 \text { años). }\end{array}$ \\
\hline Percival"1" & 12 años & $?$ & 2 años & $\begin{array}{l}\text { A io; } 5 \text { años de edad comenzaron a } \\
\text { aparecer las verrugas degenerativas. La } \\
\text { radioterapia produjo mejor efecto que } \\
\text { el radium. }\end{array}$ \\
\hline Alyarez 1 & 10 años & negativo & 2 años & $\begin{array}{l}\text { Llegó a cubrir todo el cuerpo. Múltiples } \\
\text { tumoreitos verrugoideos que se ulcera- } \\
\text { ron. Uno provocó la péndida de un ojo. } \\
\text { Evolución de fonma destructiva y mutl- } \\
\text { lante. }\end{array}$ \\
\hline
\end{tabular}


E] tratamiento se reduce a indicaciones profilácticas:

1) Evitar al máximo la exposición a la luz por el uso, en las mujeres, do grandes sombreros y de cosméticos. Lociones protectoras oclusivas a base de ácido tánico al $2 \%$ o de ácido para-aminotenzeico al $15 \%$ en una base no grasa. No debe usarse quinina por su acción fotosensibilizante. Puede usarse en esm tos casos la siguiente loción, que recomiendan Schwartz y Peck" para prevenir las quemaduras del sol: ácido isobutil-p-aminobenzoico $5 \%$, alcohol $60 \%$, glicerina $10 \%$ y agua $25 \%$; la cual puede ver adicionlada de ácido tánico al $2 \%$, para simular el tostado y aumentiar la acción protectora.

2) Las excreciones verrugowas y los epiteliomas deben ser extirpados apenas aparecen. Las ulceraciones se cubren con -ulfas o penicilina en forma de polvos : de ungüientos.

Dominga H. L. -5 años de edad.

Antecedentes hereditarios. - Padre de 28 años, sano. Maủre de 22 años, sana. No hay consanguinidad. Un tío epiléptico y otro tuterculoso que habría convivido con la chica. Un solo hermano de das años de edad, sano.

Antecedentes personales. - Nacida de paxto cutéctco, de término. Pesó al nacer $4,0000 \mathrm{~g}$. Alimentada al pecho exclustro hasta los 6 meses de edad. Después mixta con sopas y purés. Destete definitivo al año de edad. Ha sido sana. Desde el año y medio de edad ha sido criada por la abuela. Habitan en un rancho en las afueras de la ciudad. Parece no haber rectbido una alimentación carenciada.

Consultó el 8 de mayo de 1950, porque desde hacía 2 meses le había aparecido una pequeña tumoractón en Ia cara, que fué aumentando de volumen y que ziltimamerte ha sangrado con frecuencia. Al constatar que se trata de un cuadro clínico de xeroderma pigmentosum, se interroga a la abuela y a la madre, quienes no habían dado mayor importancia ni atribuído valor alguno a los signos que se describen más adelante. Sólo se Iogra obtener que a $\operatorname{los} 2$ años de edad aparecieron gran cantidad de manchas café a $\mathrm{mi}$ vel del cuello, cara y extremilades, que han ifo en aumento. Desde hace más 0 menos 3 meses notan discreta fotofobia.

Examen físico. - Peso: 16,000. Talla: 103 cm. Buen estado general. Pelo rubio, casi pa- j120, piel blanca, ojos de color café claro. Faringe libre. Amígdalas de tamaño medlano.

Piel: Las manifestaciones cutáneas se ublcan de preferencia en las partes descubiertas del cuerpo. En orden de intensidad se toman la cara, manos, antebrazos, cuello y extremidides inferiores. Escasos elementos en el resto del cuerpo. Cara algo vultuosa, mecianamente hiperhémica, con gran cantidad de manchas pigmentarias muy pequeñas, semejantes a pecas y manchas leucodérmtcas entremezcladas, que le dan a la piel un aspecto jaspeado. Telangectasias difusas, espectalmente a nivel de las mejillas. Al tacto marcada impresión de aspereza. Encima de la ceja derecha elemento lenticular, costroso y descamativo. Al lado del surco geniano de-

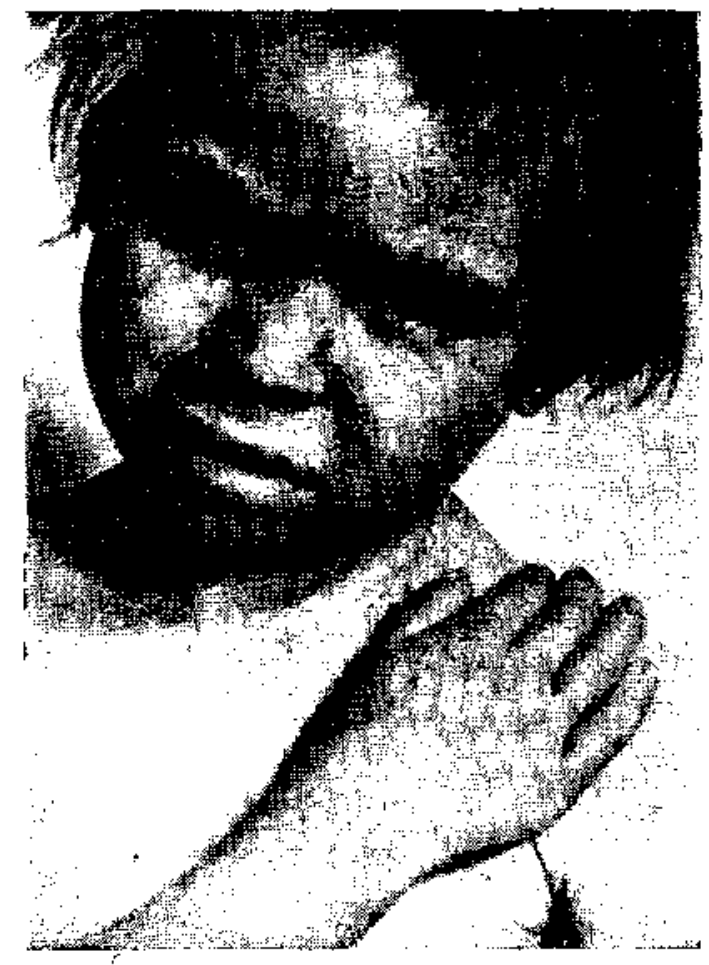

recho, inmediatamente por encima del labio, una tumoración verrugosa del tamaño de una nuez pequeña, cubierta de una costra gruesa, hemática. Labios gruesos, Jigeramente edematosos. Rinitis vestibular y coriza mucoso. Cuello: el mismo aspecto, dejando una zona de piel submentoniana libre (donde no recibe allrectamente 1a luz solar). Fxtremidades superiores: piel seca, áspera, eritematosa, intensamente plgmentada $y$ algo edematosa con ligera predomlnancia de los elementos en la cara de extensión. Hay manchas leucodérmicas y pequeños lentigos. 
Exćmenes practicados. - Oftalmológico: Bléfaro-conjuntjvitis crónica. Fondo de ojo normal. Hipermetropía y astigmatísmo. Neurológico: Probable defliciencia mental. No fué posible ahondar el examen por imhibición y falta de cooperación de la chica. Mantoux 1 x 1,000 positivo (14 $\mathrm{mm}$ ). Radioscopia de tórax negativa. Examen de orina negativo, como también las porfirinas. Colesterinemia: $1,35 \mathrm{~g}$ o/oo. Radiografia de los huesos del carpo: edad ósea normal (5 años). Reacclón de Kahn negativa. Glucemia: 0,84 g $0 / 00$. Hemograma: nada de especial. Examen coprológico: huevos de ascaris y tenias.

\section{Comentario.}

Presenta el cuadro clásico del xeroilerma pigmentosum, aparentemente entranto a la tercera elapa, que señalamos al comienzo de este relato. Hay que hazer notur do cómo el relativo abandono y la incultura del medic en que se ha descrrollado esta chica, hizo pasat, sin darlos importancia para sus padres y guardadora, los primeros períodos evolutivos de la enfermedad que, sólo el interrogatoric cuidadoso logró establecer. Otro punto que deseo destacar es que si bien no ha habido consanguinilad, que por lo demás es sólo constante en el $25 \%$ de los casos, existe el antocedento do epilepsia en un tío. Este antecedente lo bemos encontrado con relativa frecucncia en las historias de otras dermatosis on que se invoca la consauguinidad, como es la ictiosis. En efecto. on los numerosos casos que hemos observado basta la fecha, he encontrado coll triple frecuencia el de epilepsia que ol de consauguinidad. No obstante. los tratados cláricos no mencjoman el antecedunte de epilepsia entre los ascendientís del icliósico.

Actualmunte estamos investigando en esta enfernita la resesencia de porfirinus, que se suclen encertrar on los casoi de hipersensibilidad a la luz, en parte, con el fin de intentar algo más que el tratamiento preventivo, como sería el emplear el ácido pantoténico por sn probable intrivención on el metabolismo do los pigmentos tetrapirrólicos ${ }^{5}$ y el ácido fólico ${ }^{B}$. En un examen do orina no se encontraron porfirinas: pero vamos a esperar un día de sol intonso para exponer a la chica y en seguida practicar un nuevo examen, antes de concluir en la ausencia de eliminación de estos pigmentos.

Además, practicaremos una biopsia del tumor verrugoso para tratarlo on seguida de acnerdo con los técnicos del Instituto del Radium (1).

Más adelante comunicaré la evolución de esta enfermita, que espero controlar ininterrumpidamente, como también a su hermano, en ol cual también podría aparecer el cuadro.

\section{Resumen.}

So trata de una chica de 5 años de edad, con un cuadro típico de xeroderma pigmentcsum acompañado de cierto grado de deficiencia mental. No bay antecedentes de consanguinidad, pero sí de epilepsia; hecho este últino qu al autor recalca por haberlo encontrado con mayor frecuencia que el primero en nuinerosos casos de dermatosis congénitas (ictiosis), en que los tratados sólo señalan la frecuencia de la consanguinidad.

\section{Summary.}

The author reports the case of a 5 year old girl with the characteristic signs of xeroderma pigmentosulit with a curtain degres of mental deficiency. There was no history of consanguinity, althongh there was a positive one for epilepsy. The author stresses this becalse he has found this association with greater frequency than consanguinity in several cases of congenital dermatosis (ictiosis) in which the classical authors only mention the frecuency of consanguinity.

(1) La biopsia dió por resultadio un epitelioma. 


\section{Referencias.}

1.-ALVAREZ, G. - Xeroderma pigmentosum con degeneración epiteliomatosa. Arch. Argentinos de Ped. 30: 139, 1948.

2.-BiEIISARIO, J. - Two cases of Xeroderma Pizmentosum occurring in the same family. M. J. Australia 2: 148, 1935.

3.-BELL, E. T. - Xeroderma Pigmentosum. A. J. Cancer 30: 574, 1937.

4.-BLACKMAR, F. B. - Xeroderma Ptgmentosum. A. J. of Opthalmology 14: 884, 1931.

5.-BORDA y cols. - Porfiria ampollar $y$ erosiva del adulto. Prensa M. Argentina 37: 697,1850 .

6.-CASTEX, M. - Dos casos de porfirla cutánea $\mathrm{y}$ abdominal tratados con ácido fólico. Pr. M. Argentina 35: 907, 1948.

7.-FOLdvaRI, F. - Xeroderma Pigmentosum. Acta Dermat. 15: 253, 1934.
8.-LYNCH, F. W. - Xeroderma Pigmentosum. A study in sensitivity to light. Arch. Dermat. and Syph. 29: 858, 1934.

9.-MORAL.FS, M. - Tres casos de Xeroderma Pigmentosum. Arch. del Hosp. R. del Río 1: 19, 1930.

10.-PERCIVAL, G. H. - Xeroderma Pigmentosum Edinburgh M. J. I14: 1939.

11. SCHWARTZ and PECK. - Cosmeties and Dermatitis. New York, Paul B. Hoeber, 1947, pág. 144.

12. SEPULVEDA R. DARIO. - Errores frecuentes en dermatologia infantil. Por publicarse.

13.-SILBHRSTEN, A. G. - Xeroderma Pigmentosum with Mental Deficiency. A. J. D. of Children 55: 784, 1938.

14.-ZIENKIEWICZ, J. - Xeroderma Pigmentosum. Pedjatrja Polska 17; 167, 1937 (Cit. A. J. D. of Ch. 56: 932, 1938). 